\title{
Did the perpetrator have a gun, or did he not? - Children's false memories and the developmental reversal phenomenon
}

\section{Faculty of Psychology and Neuroscience}

Supervisor: Henry Otgaar

Cohort: 2012

\section{Lisanne Schreurs}

Lisanne Schreurs is currently in her third and last year of the Bachelor Psychology at Maastricht University. For their MaRBLe project, Sanne and Lisanne worked with Henry Otgaar on this study about false memories. Next year, she wants to do a forensic psychology master. Besides her courses and MaRBLe, she is a student assistant, student tutor and outside university she works at a fashion store.
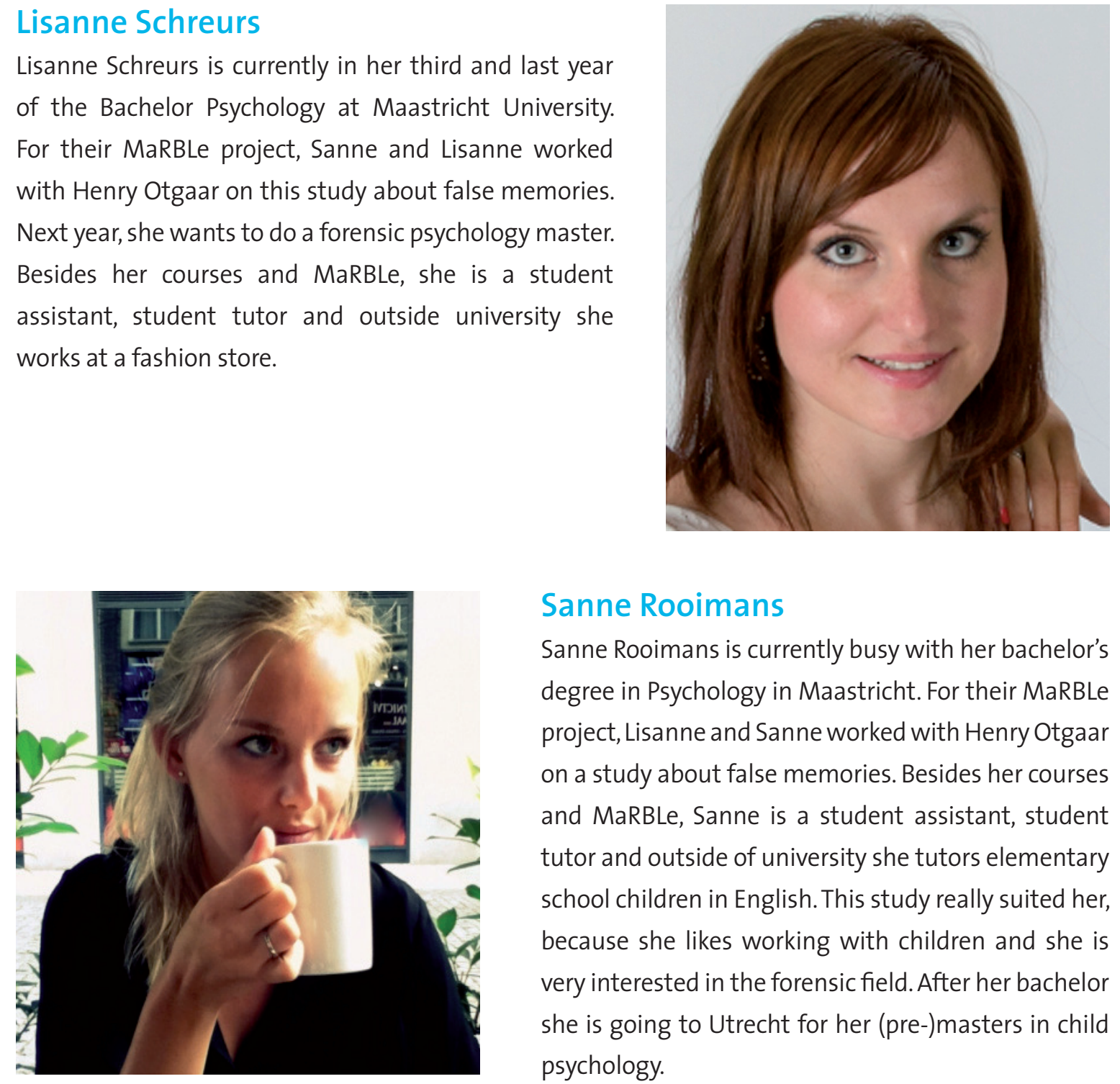

\section{Sanne Rooimans}

Sanne Rooimans is currently busy with her bachelor's degree in Psychology in Maastricht. For their MaRBLe project, Lisanne and Sanne worked with Henry Otgaar on a study about false memories. Besides her courses and MaRBLe, Sanne is a student assistant, student tutor and outside of university she tutors elementary school children in English. This study really suited her, because she likes working with children and she is very interested in the forensic field. After her bachelor she is going to Utrecht for her (pre-)masters in child psychology. 
Developmental reversal in false memories is the counterintuitive phenomenon that older children and adults are more prone to developing false memories than younger children and that false memories increase with age. The current paper discusses the theories behind this phenomenon and the paradigms that can be used to investigate it. Not only age is a prominent factor in the development of false memories. The role of emotional load of the falsely remembered words will also be investigated.

\section{False memories}

After a number of cases of people suddenly recovering false memories of childhood sexual abuse, the false memory syndrome was defined. The term false memory syndrome was created in 1992 by the False Memory Syndrome Foundation (FMSF). It describes a condition in which a person has incorrect memories that he or she strongly believes in (Brainerd \& Reyna, 2005). During the late 1990s, multiple psychiatrists and psychologists were successfully sued or reached an out-of-court settlement for implanting false memories of horrible childhood events, including sexual abuse, incest and satanic ritual abuse (Brainerd \& Reyna, 2005).

A false memory is a memory distortion of an event or detail that has never happened (Loftus, 1997). One well-known case in which false memories played a significant role took place in the small Dutch town Oude Pekela. It all started when a mother found blood in the underwear of her 4-year-old son. As she suspected sexual abuse, her son and other children at the school were interrogated (van der Hart, Boon, \& Heijtmajer Jansen, 1997). After suggestive interviews with many of children, the police believed that they had been sexually abused by people in clown suits. The children were repeatedly interviewed and other suggestive techniques included repeated questioning within interviews, the use of threats and rewards and other techniques to put the children at ease and to facilitate disclosure. Yet, there was no physical evidence of sexual abuse. Years later, researchers and the police concluded that suggestive interviewing must have caused the children to develop false memories. Suggestive interviewing leads to the creation of more false memories (Bruck \& Ceci, 1999).

The topic of false memories is an important one: if we are so susceptible to developing false memories, how do we know what really happened and what did not? False memories could have grave consequences. Indeed, people have been innocently convicted due to statements based on false memory reports (Loftus, 2003). 


\section{Developmental Reversal}

The memories of young children, older children, and adults are all susceptible to distortion. Until recently, researchers reasoned that young children compared to older children and adults are most susceptible to false memories (Ceci \& Bruck, 1993). But is this claim of developmental decline in sensitivity to false memories correct? Previous research has shown that older children and adults develop more false memories than younger children. This phenomenon is called developmental reversal (Brainerd \& Reyna, 2005).

Different theories have been proposed to explain the nature of false memories. One of these is the Fuzzy Trace Theory (Brainerd \& Reyna, 2002). According to Fuzzy Trace Theory, experiences are stored in two parallel traces:verbatim traces and gist traces. Verbatim traces are episodic representations of experienced events and refer to the encoding of subjective experiences and details of these experiences. Gist traces are the episodic interpretations of concepts that have been retrieved from memory, like underlying meanings, relations and patterns. For example, if you hear the word "horse", a gist trace would be about the underlying details you know about horses (e.g., horses have hooves, horses eat hay) and a verbatim trace would be the memory of the word "horse". There is one difference in the gist and verbatim representations of true memories and false memories. True memories are supported both by gist and verbatim representations while false memories are supported by gist representations and suppressed by verbatim representations. False memories are formed by gist processes, when verbatim processes are not present, or not strong enough to suppress the false memories (Brainerd \& Reyna, 2005).

Although young children already have the ability to form gist and verbatim traces, this ability improves with age. Therefore, adults have better gist memory than children. Because adults have more gist traces "in stock", and false memories are created by gist processes, false memories ought to develop more easily in adults relative to children, a phenomenon called developmental reversal. Fuzzy Trace Theory therefore predicts the developmental reversal (Brainerd \& Reyna, 2005).

Another false memory theory, Associative-Activation Theory, states that false memories are a product of associative-activation processes. The Associative Activation Theory relies on a direct activation system instead of a more indirect one as by the Fuzzy Trace Theory (Howe, Wimmer, Gagnon, \& Plumpton, 2009). Children's false memories, like those of adults, should increase when associative strength increases between the list items and the critical lure (Howe, 2007). Like the Fuzzy Trace Theory, Associative-Activation Theory predicts that false memories increase with age. Knowledge increases through the process of aging, as do the connections and links between all available representational concepts. As the network of connections grows, associative activation among elements increases 
and the recall of false events is more likely to happen. Increases in false memories with age are related to increases in the direct activation of associative connections with age (Howe, Wimmer, Gagnon \& Plumpton, 2009). Both the Fuzzy Trace Theory and the AssociativeActivation Theory predict developmental reversal.

\section{DRM paradigm}

The DRM paradigm is often used to investigate the developmental reversal phenomenon, because sensitivity for the so called DRM illusion increases with age (Brainerd, Forrest, Karibian, \& Reyna, 2006). The DRM paradigm is a procedure which involves presenting a list of semantically related words (e.g., blood, hospital, illness) of which participants have to memorize a wordlist. After a distraction task, they are presented with another wordlist and have to state whether they have previously heard the word or not. The memory of hearing a word which has not been presented is a DRM illusion, which is also called a false memory. Howe (2006) found that, consistent with other DRM research (Brainerd, Reyna, \& Forrest, 2002; Howe, 2005, 2006; Howe, Cicchetti, Toth, \& Cerrito, 2004; Howe, Gagnon, \& Thouas, 2008), children from three different age groups produce true and false memories and that the number of both true and false memories increase significantly with age. The developmental reversal phenomenon in the DRM paradigm can be explained with the Fuzzy Trace Theory. The probability that people will store the type of gist memories that cause the false memory increases with age, according to this theory. Processing gist traces allows for linking different words with each other. The DRM illusion makes it difficult to suppress false memories through retrieving verbatim traces. The Associative-Activation Theory explains that false memories in the DRM task arise from associative activation which increases with age (Meade, Jason, Balota, \& Roediger, 2007). Both the Fuzzy Trace Theory and the Associative-Activation Theory can explain developmental reversal in the DRM illusion. Further research is necessary to conclude which theory explains the phenomenon best.

\section{Misinformation paradigm}

Another paradigm that can be used to investigate false memories and developmental reversal is the misinformation paradigm. In the misinformation paradigm, the participant observes an event before receiving misinformation. Next, the memory for the observed event is tested for distortions due to the misinformation. This can be done using a recognition task or by asking the participants what they observed. Roediger, Jacoby, and 
McDermott (1996) found a robust misinformation effect in recall and concluded that false memories may arise through retrieval of the misinformation. The misinformation paradigm can be used to elicit developmental reversal. False memories in the misinformation task are caused by retrieval of verbatim traces as well as by retrieval of gist traces. However, only the retrieval of verbatim traces leads to a decrease in correct answers. The misinformation adds (verbatim) traces representing the false information to the traces for correct information. (Brainerd \& Reyna, 1998). If the misinformation verbatim trace is retrieved when it is tested, the answer will be wrong. The retrieval of verbatim and gist traces increases with age which leads to an increase in false memories (Brainerd \& Reyna, 1998).

\section{Emotion and false memories}

From previously discussed studies it seems clear that there is developmental reversal in false memories. However, there is not much research yet on the influence of the emotional load of words or events. The aim of our own study was to replicate prior findings with regard to developmental reversal and the emotional significance of falsely remembered words. We hypothesized that older children would be more susceptible to false memories than younger children and that more false memories would develop for negative rather than neutral words.

Children from two age groups took part in the study: 22 of the children were between 7 and 8 years old and 25 children were between 10 and 11 years old. For half of the children, the experiment started with a robbery video. The fictional scene took place in a bar where a few people were drinking, eating and playing darts when suddenly a robber entered the room. He took all wallets and fled with his accomplice who was standing at the doorpost.

The children were asked to watch the video closely and to remember as many details as possible. Afterwards, they performed a distraction task for three minutes. With this task we distracted participants' attention from the actual task, so they could not repeat the words or scene from the video in their head.

After seeing the video about the robbery and performing the distraction task, the children listened to a statement given by a witness. Half of the children received a statement including misinformation (misinformation condition) while the other half did not (no misinformation condition). In the misinformation condition, the witness gave false information about, for example, the robber having a pistol or people eating from plates while they were actually eating from napkins. 
After performing another filler task, a recognition task followed. This recognition task included a wordlist consisting of 68 words. The words represented objects, like 'black coat' or 'dartboard' which participants could have seen in the video. Besides these words there were so called 'related words' which were not present in the video, like 'cash machine', non-related words like 'horse' or 'inflatable swimming pool', and critical lure words like 'pistol' in the wordlist. The critical lure words in the list were the words that were taken as indicative of a false memory. Dalton and Daneman (2006) previously found that participants were susceptible to misleading suggestions about central features which are directly related to the recognition task, although to a lesser extent than they were susceptible to misleading suggestions about peripheral features. This is why we divided the words into two categories, concerning the relation of the word to the robbery video. The central words were directly related to the video (e.g., wallet, perpetrator) and the peripheral words were not directly related (e.g., cash machine, $€$ sign).

Furthermore, children listened to a DRM word list and had to remember as many words as they could. After another distraction task, they listened to another word list and stated whether they had heard the words before or not. They said "yes" out loud when they recognized the word and "no" when they did not. We distinguished 4 different answer possibilities. If their answer was correct, it was called a 'hit'. If they claimed to have heard a word that was not in the wordlist, the word could be classified in one of the following groups: 'relevant word', 'non relevant word' or 'false memory/critical lure'. For example, if the words in the wordlist were 'baker', 'flour' and 'grain', then the false memory word would be 'bread'. Relevant words were not present in the wordlist, but were relevant for the word categories (e.g., syrup is relevant for the category "sweet"). Non relevant words had absolutely nothing to do with the words in the word list (e.g., shelf is not relevant for any of the categories).

Next to the classification described above, we divided words into neutral and negative categories. Negative words had negative emotional load, such as 'scared' or 'murderer'. Neutral words, for example 'window frame' or 'dough', were emotionally neutral. Previous research (Otgaar, Candel, \& Merckelbach, 2008) showed that children develop are more likely to develop false memories about items with a negative connotation, compared to emotionally neutral items.

Results showed that for the DRM paradigm, the amount of critical lures (false memories) was significantly different between the two age groups. Older children developed false memories at a higher rate in the DRM task (6.6 false memories on average) compared to younger children ( 4.6 false memories on average; see Figure 2). This is in line with the findings of Howe (2007) who studied false memories with the DRM paradigm in 8 to 

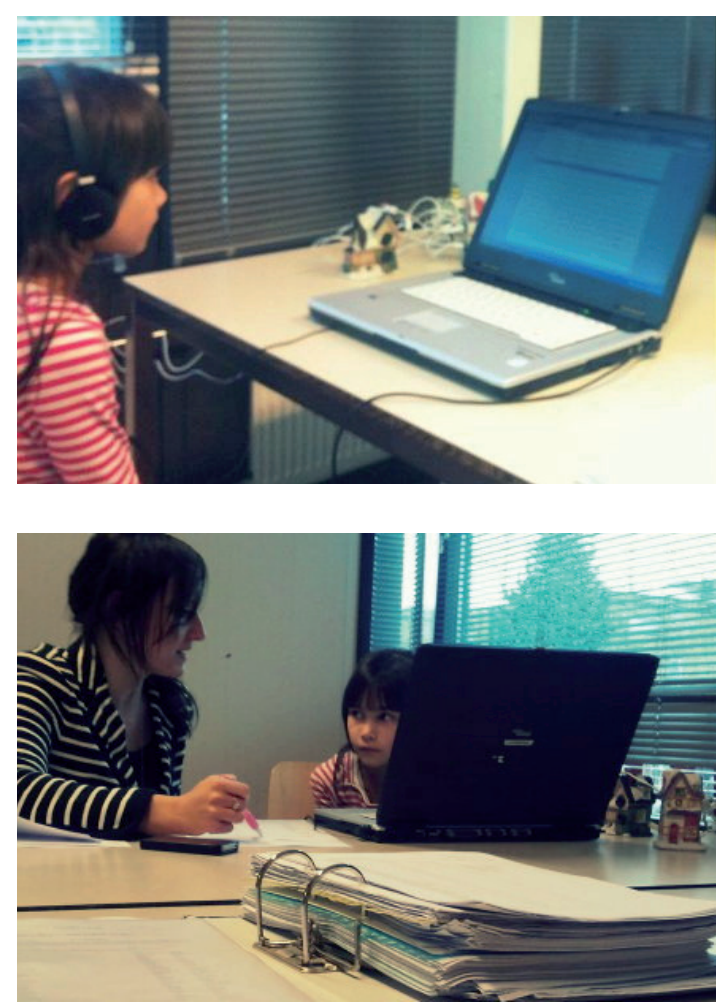

13-year-old children. According to Brainerd, Reyna \& Zember (2011) 55 experiments have used the DRM illusion to provide clear evidence that false memories increase from early childhood onward.

We also hypothesized that critical lures would be more common in the case of negative words/wordlists compared to neutral wordlists. This hypothesis was supported by our data. This is consistent with results obtained by Otgaar et al. (2008). In this study, the negative narratives elicited more false memories than the neutral narratives in 7 year olds. In the legal area, children are often interviewed about negative events. Given the current results, it should be considered that false memories might occur more often in this context.

Furthermore, our participants were expected to develop fewer false memories for the central words in the recognition task. This hypothesis was also supported. Just as Luna and Migueles (2009), we found that eyewitness testimonies were easily distorted in peripheral aspects, but that mistakes with regard to central information were less common. Luna and Migueles explained that the cognitive system has a limited attentional capacity and a greater information capacity for central elements which make it easier to process the more important central information. However, when misinformation was introduced, just as in our tasks, errors in central information occurred more frequently.

For the recognition test, no significant results were found for differences between age groups, looking at false memories (see Figure 3). This was unexpected because significant developmental reversal results were actually visible for the DRM task. One possible explanation is the small difference in age between our two groups of children.

To recap, we found that older children had better memory for correct words than the younger children. Although young children have the ability to form both gist and verbatim traces, this ability improves with age. Therefore, adults and older children have better gist memory than younger children, according to the Fuzzy Trace Theory (Brainerd \& Reyna, 2005). This is why we expected older children to have better true memory than the younger children, which was indeed the case. 


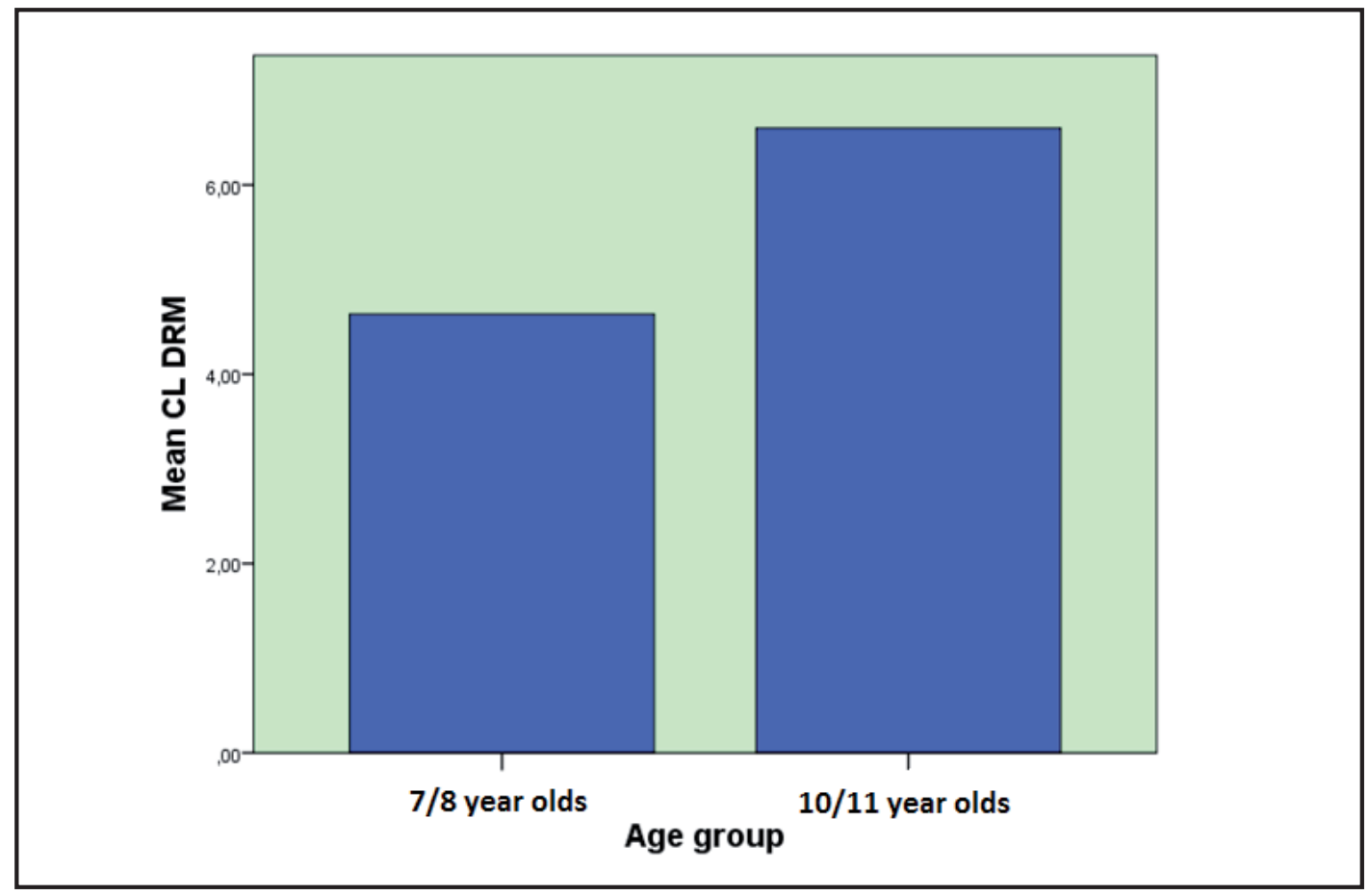

Figure 2: Mean number of critical lures (false memories) in the DRM task

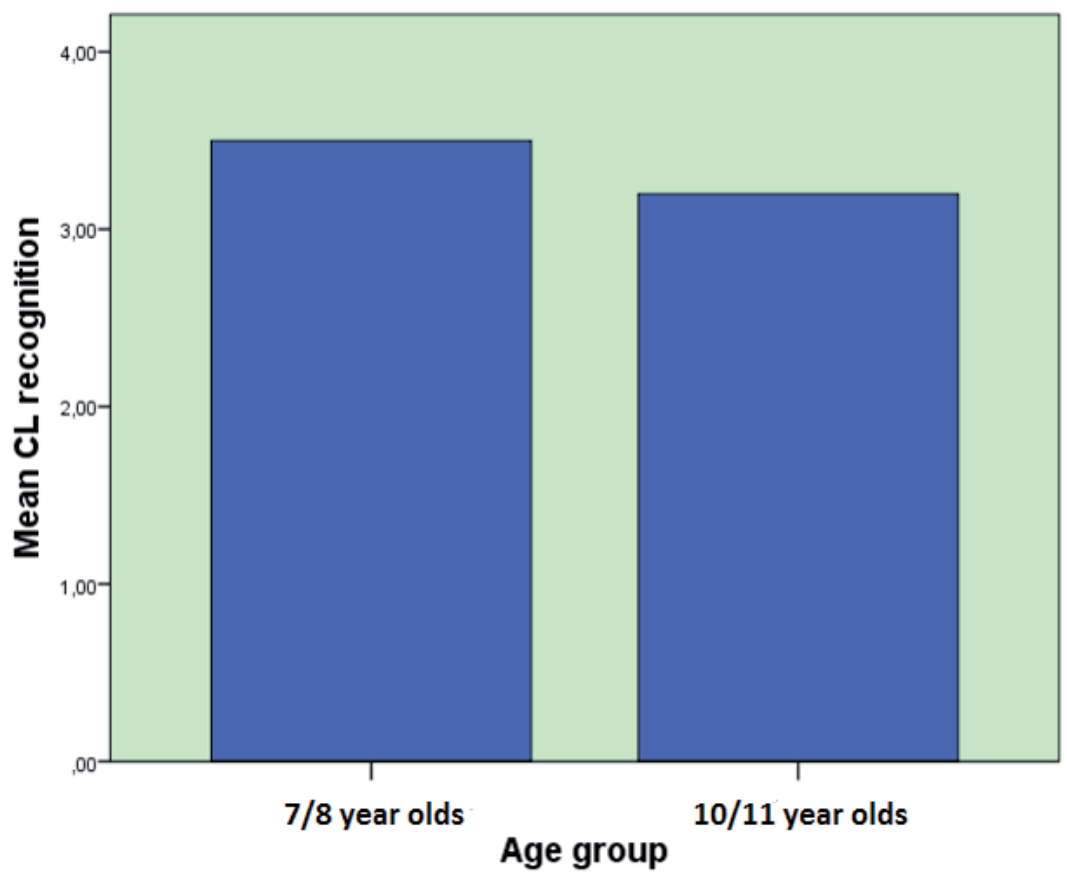

Figure 3: Mean critical lures (false memories) for the recognition task 


\section{Conclusions}

As previous research suggested, we can conclude that using the DRM paradigm, older children are indeed more susceptible to false memories than younger children. Accordingly, older children's witness statements are more likely to be infected with false memories than those of younger children. Thus, the assumption that juries should give more weight to adults' versions of events may not be as plausible as generally assumed. This must be taken into account when interviewing children in legal situations to avoid convictions based on false memories. But not only age influences the development of false memories, the emotional load of the event is important as well. Our results provide supporting evidence for the fact that false memories are more likely to arise when the memories concern negative events which is usually the case in court.

\section{Glossary}

Associative-Activation Theory: states that false memories are a product of associativeactivation processes. Knowledge increases through the process of aging, as do the connections and links between all available representational concepts. As the network of connections grows, associative activation among elements increases and the recall of false events is more likely to happen.

Developmental reversal (in the context of false memories): the counterintuitive phenomenon that false memories increase with age

DRM illusion: the memory of hearing a word that was not presented in the DRM word list. DRM paradigm: a procedure which involves presenting a list of semantically related words of which participants have to memorize as many as possible. After a distraction task, they are presented with another wordlist and have to state whether they have previously heard the word before or not.

False memory: a memory distortion of an event or detail that has never happened or does not correspond to truth and reality.

Fuzzy-Trace Theory: states that experiences are stored in two parallel traces: verbatim traces and gist traces. Verbatim traces are episodic representations of experienced items events and refer to the encoding of subjective experiences. Gist traces are the episodic interpretations of concepts that have been retrieved from memory. False memories are formed by gist processes.

Misinformation effect: exposure to misinformation between the encoding and recall of information leads to distortions in memory.

Misinformation paradigm: In this paradigm, participants are presented with misinformation after witnessing an event. Hereafter, the memory of the observed event is tested for distortions due to the misinformation. These memory distortions are false memories. 


\section{References}

Brainerd, C., Forrest, J., Karibian, D., \& Reyna, V. (2006). Development of the false-memory illusion. Developmental Psychology, 42, 962-979.

Brainerd, C., \& Reyna, V. (1998). Fuzzy-Trace Theory and children's false memories. Journal of Experimental Child Psychology, 71, 81-129.

Brainerd, C., \& Reyna, V. (2002). Fuzzy-Trace Theory and false memory. American Psychological Society, 11, 164-169.

Brainerd, C., \& Reyna, V. (2005). The science of false memory. New York: Oxford University Press.

Brainerd, C., Reyna, V.,\& Zember, E. (2011). Theoretical and forensic implications of developmental studies of the DRM illusion. Journal of Experimental Psychology: Learning, Memory, and Cognition, 39, 365-380.

Bruck, M., \& Ceci, S. (1999). The suggestibility of children's memory. Annual Review of Psychology, 50, 419-439.

Ceci, S. J., \& Bruck, M. (1993). Suggestibility of the child witness: A historical review and synthesis. Psychological Bulletin, 113, 403-439

Dalton, A., \& Daneman, M. (2006). Social suggestibility to central and peripheral misinformation. Memory, 14, 486-501.

Howe, M. L. (2005). Children (but not adults) can inhibit false memories. Psychological Science, 16, 927-931

Howe, M. (2006). Developmentally invariant dissociations in children's true and falsememories: Not all relatedness is created equal. Child Development, 77, 1112-1123.

Howe, M. (2007). Children's emotional false memories. Psychological Science, 18, 856-860.

Howe, M. L., Cicchetti, D., Toth, S. L., \& Cerrito, B. M. (2004). True and false memories in maltreated children. Child Development, 75, 1402-1417

Howe, M. L., Gagnon, N., \& Thouas, L. (2008). Development of false memories in bilingual children and adults. Journal of Memory and Language, 58, 669-681.

Howe, M., Wimmer, M., Gagnon, N., \& Plumpton, S. (2009). An Associative-Activation Theory of children's and adults' memory illusions. Journal of Memory and Language, 60, 229-251.

Loftus, E. (1997). Creating false memory. Scientific American, 277, 70-75.

Loftus, E. (2003). Make-believe memories. American Psychologist, 58, 867-873.

Luna, K., \& Migueles, M. (2009). Acceptance and confidence of central and peripheral misinformation. Spanish Journal of Psychology, 12, 405-413.

Meade, M., Watson, J., Balota, D., \& Roediger, H. (2007). The roles of spreading activation and retrieval mode in producing false recognition in the DRM paradigm. Journal of Memory and Language, 56, 305-320. 
Otgaar, H., Candel, l., \& Merckelbach, H. (2008). Children's false memories: Easier to elecit for a negative than for a neutral event. Acta Psychologica, 128, 350-354.

Roediger, H., Jacoby, J., \& McDermott, K. (1996). Misinformation effects in recall: Creating false memories through repeated retrieval. Journal of Memory and Laguage, 35, 300-318. Van der Hart, O., Boon, S., \& Haijtmajer Jansen, O. (1997). Ritual abuse in European countries: A clinical's perspective. In G. A. Fraser (Ed.), The dilemma of ritual abuse: Cautions and guides for therapists (pp. 137-164). Washington DC: American Psychiatric Press. 